\title{
eJRIEPS
}

Ejournal de la recherche sur l'intervention en éducation physique et sport

47 | 2020

Varia

\section{Les sources de connaissance privilégiées par les entraineurs belges francophones}

Sources of knowledge favoured by Francophone Belgian coaches

\section{Pierre Trudel et Marc Cloes}

\section{(2) OpenEdition}

Journals

Édition électronique

URL : http://journals.openedition.org/ejrieps/5222

DOI : 10.4000/ejrieps.5222

ISSN : 2105-0821

Éditeur

ELLIADD

Référence électronique

Pierre Trudel et Marc Cloes, « Les sources de connaissance privilégiées par les entraineurs belges francophones », eJRIEPS [En ligne], 47 | 2020, mis en ligne le 01 juillet 2020, consulté le 21 novembre 2020. URL : http://journals.openedition.org/ejrieps/5222 ; DOI : https://doi.org/10.4000/ejrieps.5222

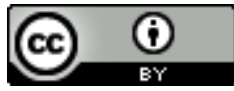

La revue eJRIEPS est mise à disposition selon les termes de la Creative Commons Attribution 4.0 International License. 


\section{Les sources de connaissance privilégiées par les entraineurs belges francophones}

\section{Pierre Trudel ${ }^{*} \&$ Marc Cloes**}

*École des sciences de l'activité physique, Université d'Ottawa, Ontario, Canada.

** Département des Sciences de la motricité, Université de Liège, Belgique.

\section{Résumé}

Sur la base des informations fournies par 85 entraineurs ${ }^{1}$ sportifs belges francophones, nous avons identifié les sources de connaissance sur lesquelles ils se sont le plus appuyés pour acquérir leur niveau de compétence actuel dans cinq tâches caractéristiques de leur activité et celles qu'ils auraient souhaité pouvoir bénéficier dans la même perspective. Les résultats corroborent ce qui a été montré dans plusieurs pays. Ils mettent par ailleurs en évidence pour la première fois que la manière d'acquérir les connaissances diffère selon les types de tâches concernées. L'étude permet d'identifier de nombreuses pistes de recherche potentielles de même que des perspectives intéressantes pour les responsables de formations d'entraineurs.

Mots clés : Formation; Entraineurs; Connaissances acquises

Sources of knowledge favoured by Francophone Belgian coaches

Summary

Based on the answers provided by 85 sport coaches from the French speaking part of Belgium, we identified knowledge on which they built their actual level of competence in five specific tasks of their coaching activities as well as those that they would have appreciated to acquire in the same perspective. Findings confirm what has been underlined in several countries. However, they emphasize for the first time that the way to acquire knowledge differs according to the kind of task envisaged. The study allows identifying several potential research tracks as well as interesting perspectives for coach education managers.

Key words: Coach Education; Content knowledge

1 L'usage du masculin vise uniquement à alléger le texte. Les termes utilisés renvoient autant aux hommes qu'aux femmes 


\section{Introduction}

Quel que soit leur âge, la pratique sportive joue un rôle important dans la vie des gens. Lorsque les conditions sont favorables, elle facilite, entre autres, le développement moteur, le développement social et l'atteinte d'objectifs de performances sportives (Beaudoin, Callary, \& Trudeau, 2015; Côté, Lidor, \& Hackfort, 2009; Young \& Callary, 2018). L'entraineur est souvent cité comme la personne clé pour la préparation des conditions d'une pratique sportive saine et optimale (Hamilton \& LaVoi, 2019). Aussi, de nombreux pays ont mis en place des programmes de formation. Par exemple, depuis 1975, le Canada a institué le Programme National de Certification des Entraineurs (PNCE), lequel a subi d'importantes modifications au fil des années, incluant l'intégration de principes d'apprentissage dits constructivistes (Culver, Werther, \& Trudel, 2019). Pour se familiariser avec le type de programmes offerts dans différents pays, nous suggérons de consulter la section 'Insight' de l'International Sport Coaching Journal. Bien qu'il existe certaines différences dans la structure de ces programmes, ils ont souvent deux points en commun. Premièrement, le contenu est divisé en modules destinés à être enseignés dans le cadre de cours dispensés hors des périodes d'activités professionnelles. Cette manière de précéder semble le format idéal considérant que le temps à investir et les coûts financiers sont souvent des barrières à la participation des entraineurs (Araya, Bennie, \& O'Connor, 2015; Nash \& Sproule, 2012; Vargas-Tonsing, 2007). Deuxièmement, ces programmes permettent aux entraineurs de recevoir une certification, laquelle est souvent nécessaire pour diriger un groupe sportif à un niveau donné et/ou obtenir des subventions (Callary, Culver, Werthner, \& Bales, 2014). Lorsqu'on les interroge sur leur niveau de satisfaction à propos de ces programmes, les entraineurs ont souvent des opinions divergentes. II ressort clairement que ces programmes ne sont qu'une source de connaissance parmi d'autres (Piggott, 2012). Plusieurs études sur les sources de connaissance des entraineurs ont été publiées depuis le début du $21^{\text {ème }}$ siècle. En additionnant les revues de littérature de Cushion et al. (2010) pour la période de 1999-2008 et celle de He, Trudel et Culver (2018) pour la période de 2009-2016, un total de 36 articles peut ainsi être identifié. Dès lors, et tel que mentionné par Cushion et al., " on pourrait soutenir que l'étude des situations d'apprentissage des entraineurs est devenue un domaine distinct et légitime de la recherche académique » (p. 23, traduction libre).

A partir du moment où il est admis que le développement des entraineurs se fait au travers de plusieurs sources de connaissance, le concept de l'apprentissage tout au long de la vie prend tout son sens (Duarte \& Culver, 2014; Lara-Bercial \& Mallett, 2016; Watts \& Cushion, 
2017). Pour les responsables des programmes de formation, cette manière de concevoir le processus par lequel les entraineurs apprennent à devenir entraineurs, n'est pas sans conséquence et amène son lot de défis (Trudel, Culver, \& Richard, 2016). En plus de promouvoir l'utilisation de nouveaux concepts reliés à l'apprentissage (Paquet \& Trudel, 2018a, b), elle doit aider les formateurs d'entraineurs à adopter des stratégies d'interventions différentes (Paquette, Trudel, Duarte, \& Cundari, 2019). Selon Jones et Wallace (2005), les chercheurs et les praticiens dans le milieu du sport sont souvent trop pressés de trouver des solutions et d'identifier les meilleures pratiques. Ces auteurs soulignent également qu'il faudrait commencer par une phase visant à «chercher des connaissances pour comprendre [knowledge-for-understanding] ce qui donnerait une meilleure compréhension du processus complexe de coaching. A leur tour, ces connaissances offriraient une base plus sûre sur laquelle les projets de connaissances pour l'action [knowledge-for-action] pourraient s'appuyer » (traduction libre, p. 123).

\section{Question de recherche}

Prenant en considération les points soulevés ci-dessus, le présent article porte sur une étude descriptive dont l'objectif général était d'investiguer les sources de connaissance d'entraineurs belges francophones (Fédération Wallonie-Bruxelles - FWB). Plus précisément, nous voulions répondre à la question suivante : quelles sont les principales sources de connaissance utilisées en Belgique francophone par les entraineurs de niveau récréatif ou de développement (R/D) et les entraineurs de haut niveau $(H N)$ ?

\section{Méthodologie}

\subsection{Contexte de l'étude}

En Belgique, les compétences des autorités publiques en matière de sport sont communautarisées (placées sous la responsabilité d'une des trois communautés linguistiques: néerlandophone, francophone ou germanophone - Cloes, 2012). Depuis 2012, l'Administration Générale du Sport a mis en place une vaste réforme du système de formation des entraineurs et a renforcé ses exigences tant au niveau des cours généraux (dispensés aux entraineurs de toutes les disciplines sportives) que pour les cours spécifiques (placés sous la responsabilité des fédérations devant respecter des cahiers des charges très stricts).

Avec le support de l'Administration Générale du Sport de la FWB, les fédérations de volleyball (FVWB) et de rugby (LBFR) ont joint leurs efforts pour offrir aux entraineurs belges francophones un atelier de formation portant sur 'le développement des connaissances 
chez les entraineurs'. D'une durée d'environ 120 minutes, l'atelier était animé par Pierre Trudel et le contenu inspiré de ses travaux (e.g., He et al., 2018; Milistetd, Peniza, Trudel, \& Paquette, 2018; Trudel et al., 2016; Trudel, Gilbert, \& Rodrigue, 2016). Les objectifs visés étaient (a) de discuter des différentes sources de connaissance qu'utilisent les entraineurs, (b) de faire comprendre que les entraineurs sont responsables de leur développement et devraient être proactifs et (c) de guider les entraineurs dans la rédaction d'une ébauche d'un plan de développement personnel. L'atelier a été présenté dans cinq régions de la FWB afin de limiter le déplacement des entraineurs et augmenter ainsi le taux de participation. L'atelier fut présenté à cinq reprises à des entraineurs de niveau récréatif et/ou de développement $(R / D)$ et une fois à un groupe d'entraineurs de haut niveau $(H N)$. Compte tenu du volet pratique inclus dans l'atelier, un maximum de 30 participants avait été fixé pour chaque séance. Toutes les fédérations sportives francophones avaient été invitées à inciter leurs entraineurs à participer à ces ateliers. Toutefois, la priorité était donnée aux entraineurs de volley-ball. Sur les 123 entraineurs qui ont participé aux six ateliers, 39\% étaient issus de la FVWB tandis que les autres provenaient du football (soccer), du rugby et de différents sports individuels. Le tableau I présente la distribution des fiches qui ont été concrètement recueillies et analysées (les entraineurs étaient libres de partager leurs productions).

Tableau I. Données sur la participation des entraineurs aux ateliers (n)

\begin{tabular}{|l|c|c|c|c|c|}
\hline & Volley-ball & Football & Autres & Total & Fiches analysées \\
\hline Entraineurs R/D & 40 & 18 & 32 & 90 & 66 \\
\hline Entraineurs HN & 8 & 4 & 21 & 33 & 19 \\
\hline Total & 48 & 22 & 53 & 123 & 85 \\
\hline
\end{tabular}

\subsection{Collecte des données}

Au tout début de chaque atelier, les participants étaient invités, de manière anonyme, à compléter une fiche sur les sources de connaissance qui ont le plus contribué à leur apprentissage du rôle d'entraineur. Cette fiche était une adaptation de celle utilisée par He et al. (2018) avec des entraineurs chinois de gymnastique artistique et gymnastique rythmique. La fiche se divise en sept sections (tableau II). La première section présente une liste des sources de connaissance les plus souvent mentionnées dans la littérature. L'animateur prenait quelques minutes pour s'assurer de la compréhension de chacune des sources. Par exemple, la source 'Journal/Carte réflexive' faisait référence à des moments de réflexion à l'aide d'outils spécifiques tel un journal de bord (Koh, Mallett, Camiré, \& Wang, 
2015; Kuklick, Gearity, \& Thompson, 2015) ou une carte sur laquelle il y a des questions spécifiques à répondre après une séance d'entrainement ou un match (Rodrigue \& Trudel, 2018). Pour ce qui est de 'l'expérience comme athlète' et de 'l'expérience comme entraineur', il s'agissait du vécu personnel dans chacun de ces contextes, quel que soit le niveau de participation et le nombre d'années de pratique. Par la suite, la consigne suivante était donnée :

Parmi les sources de connaissance énumérées, identifiez celles qui ont le plus contribué à qui vous êtes aujourd'hui comme entraineur en inscrivant sous la colonne 'Act' (actuel) le chiffre 1 vis-à-vis à la plus importante, 2 pour la seconde et ainsi de suite. Répétez l'exercice en inscrivant sous la colonne 'Idéal' ce que vous auriez préféré.

Cette démarche fut reprise pour cinq tâches liées au travail des entraineurs : (a) Faire la sélection de mes joueurs; (b) Développer un plan annuel d'entrainement; (c) Enseigner les habiletés techniques et tactiques; (d) Enseigner les habiletés psychologiques, et; (e) Guider mes athlètes avant, pendant et après une compétition. Ces cinq tâches ont été sélectionnées pour les raisons énumérées ci-après :

(1) Elles font partie d'une des trois dimensions du modèle de coaching de Côté, Salmela, Trudel, Baria, et Russell (1995), un modèle souvent utilisé dans les recherches sur l'entrainement sportif (Bennie \& O'Connor, 2011; Côté, 2006; Din \& Paskevich, 2013).

(2) Les entraineurs sont souvent à la recherche d'information sur ces tâches (Carter \& Bloom, 2009).

(3) L'importance de chacune de ces tâches a été mise en évidence dans la littérature scientifique. Par exemple, 'la sélection des joueurs' est une tâche difficile et parfois ingrate (Capstick \& Trudel, 2010; Roberts \& Faull, 2013). La 'planification annuelle d'entrainement' constitue une tâche complexe dont les recommandations théoriques évoluent régulièrement (Issurin, 2010; Roy, Roy, Chevrier, \& Cardinal, 2018). Enseigner 'les habiletés techniques et tactiques' représente une tâche jugée essentielle à tout bon entraineur (Bolter, Petranek, \& Dorsch, 2018), tout comme l'enseignement des 'habiletés psychologiques' (Pope et al., 2015; Thelwell, Weston, Greenlees, \& Hutchings, 2008).

(4) La qualité du travail avant/pendant/après les compétitions constitue une autre tâche importante puisque ces rendez-vous représentent des situations stressantes tant 
pour les athlètes (Kristiansen \& Roberts, 2010) que pour les entraineurs (Olusoga,

Butt, Maynard, \& Hays, 2010).

A l'issue de l'exercice, une période de 10 minutes était accordée pour que, en petits groupes, les entraineurs comparent et discutent leurs résultats. Par la suite, un modèle illustrant le développement des entraineurs (Trudel, Gilbert \& Rodrigue, 2016) était présenté et discuté. L'atelier se terminait avec un exercice où chaque entraineur était invité à construire son plan de développement personnel. Pour ce faire, l'entraineur devait d'abord choisir un maximum de cinq aspects de l'entrainement sportif pour lesquels il souhaitait acquérir des connaissances et préciser ensuite quelles seraient les sources de connaissance qu'il pourrait utiliser pour y parvenir. Afin d'encourager les entraineurs à utiliser leur plan de développement, il leur a été proposé d'envoyer, deux mois après l'atelier, une fiche décrivant ce qu'ils avaient pu réaliser.

\subsection{Analyse des données}

Dans le but d'éviter une multitude de tableaux et de chiffres, les décisions suivantes furent prises. Premièrement, nous avons limité l'analyse aux deux plus importantes sources de connaissance identifiées par les entraineurs. Ceci implique que la lecture des tableaux doit se faire de la manière suivante. Par exemple, dans le tableau II, le chiffre 64 proposé dans la première cellule signifie que 64\% (42/66) des entraineurs ont choisi 'Expérience d'athlète' comme première ou deuxième source de connaissance. La deuxième décision concerne la différence entre les chiffres pour 'Actuel' et 'Idéal'. Seules les différences de $10 \%$ et plus ont été rapportées afin de faciliter la lecture des tableaux. Une valeur positive indique que les entraineurs auraient préféré (idéalement) utiliser davantage cette source de connaissance. A l'inverse, une valeur négative signifie que les entraineurs auraient préféré que cette source de connaissance soit moins importante.

\section{Résultats}

Les données du tableau II permettent d'établir certains constats sur les principales sources de connaissance des entraineurs R/D. Premièrement, les chiffres dans les cases sous 'Actuel' (Act.) indiquent que l'expérience comme entraineur et l'expérience comme athlète sont les deux sources de connaissance qui ont le plus contribué à qui ils sont comme entraineurs ainsi que pour accomplir les cinq tâches. Deuxièmement, les chiffres dans les cases sous 'Différence' (Dif.) indiquent que, idéalement, les entraineurs aimeraient dépendre moins de leur expérience comme athlètes (bien que toujours importante) et augmenter l'acquisition de connaissances par les programmes de certification en sport et 
les conférences/séminaires. Troisièmement, l'importance des sources de connaissance peut varier considérablement d'une tâche à une autre. Par exemple, si l'expérience comme entraineur est une source de connaissance très importante (82\%) pour faire la sélection des joueurs, elle l'est beaucoup moins (50\%) pour l'enseignement des habiletés techniques et tactiques. Quatrièmement, l'accès à un mentor et discuter avec d'autres entraineurs sont considérés comme d'importantes sources de connaissance pour seulement au maximum $20 \%$ des entraineurs. Finalement, l'acquisition de connaissances par les livres/vidéos, Internet et par des outils de réflexion semble négligeable.

Les données du tableau III sur les perceptions des entraineurs HN donnent un portrait global qui ressemble à celui des entraineurs R/D. Les sources 'Expérience-entraineur' et 'Expérience-athlète' dominent. Idéalement, les entraineurs HN auraient préféré avoir à moins se reposer sur leur expérience d'athlète et sur les autres entraineurs mais acquérir davantage de connaissances par les programmes de certification et les conférences/séminaires. Cette préférence est particulièrement présente pour la tâche 'Enseigner les habiletés psychologiques', ce qui était également vrai pour les entraineurs R/D.

Tableau II. Sources de connaissance des entraineurs de niveau récréatif ou de développement $(n=66)$

\begin{tabular}{|c|c|c|c|c|c|c|c|c|c|c|c|c|c|c|c|c|c|c|}
\hline \multicolumn{19}{|c|}{ Sélection des plus importantes (i.e. deux premières) sources de connaissance actuelles (Act.) et idéales (Idéal) (\% de réponses) } \\
\hline \multirow[t]{2}{*}{$\begin{array}{c}\text { Sources de } \\
\text { connaissance }\end{array}$} & \multicolumn{3}{|c|}{$\begin{array}{l}\text { Qui suis-je } \\
\text { (entraineur) }\end{array}$} & \multicolumn{3}{|c|}{ Sélection de joueurs } & \multicolumn{3}{|c|}{$\begin{array}{c}\text { Plan annuel } \\
\text { d'entrainement }\end{array}$} & \multicolumn{3}{|c|}{$\begin{array}{c}\text { Habiletés techniques } \\
\text { et tactiques }\end{array}$} & \multicolumn{3}{|c|}{$\begin{array}{c}\text { Habiletés } \\
\text { psychologiques }\end{array}$} & \multicolumn{3}{|c|}{$\begin{array}{c}\text { Compétition } \\
\text { avant/pendant/après }\end{array}$} \\
\hline & Act. & Idéal & Dif. & Act. & Idéal & Dif. & Act. & Idéal & Dif. & Act. & Idéal & Dif. & Act. & Idéal & Dif. & Act. & Idéal & Dif. \\
\hline Expérience - athlète & 64 & 39 & -25 & 67 & 56 & -11 & 32 & 26 & & 64 & 50 & -14 & 55 & 30 & -25 & 62 & 41 & -21 \\
\hline Expérience - entraineur & 65 & 56 & & 82 & 71 & -11 & 53 & 49 & & 50 & 55 & & 68 & 56 & -12 & 67 & 68 & \\
\hline Certification en sport & 33 & 49 & 16 & 18 & 39 & 21 & 49 & 64 & 15 & 24 & 50 & 26 & 18 & 41 & 23 & 21 & 35 & 14 \\
\hline Conférences, séminaires & 12 & 23 & 11 & 5 & 8 & & 24 & 29 & & 14 & 21 & & 11 & 30 & 19 & 11 & 14 & \\
\hline Mentor & 11 & 23 & 12 & 11 & 9 & & 11 & 15 & & 23 & 14 & & 21 & 20 & & 17 & 18 & \\
\hline Autres entraineurs & 15 & 5 & -10 & 14 & 9 & & 18 & 6 & -12 & 17 & 6 & -11 & 14 & 11 & & 15 & 11 & \\
\hline Livres/Vidéos & 2 & 2 & & 2 & 3 & & 8 & 5 & & 6 & 3 & & 9 & 8 & & 5 & 3 & \\
\hline Internet & 2 & 0 & & 0 & 0 & & 3 & 2 & & 3 & 1 & & 3 & 0 & & 0 & 0 & \\
\hline Journal/Carte réflexive & 0 & 2 & & 0 & 2 & & 2 & 6 & & 0 & 0 & & 2 & 5 & & 3 & 8 & \\
\hline Autre & 0 & 0 & & 0 & 3 & & 0 & 2 & & 0 & 0 & & 0 & 0 & & 0 & 3 & \\
\hline
\end{tabular}


Tableau III. Sources de connaissance des entraineurs de haut niveau $(n=19)$

\begin{tabular}{|c|c|c|c|c|c|c|c|c|c|c|c|c|c|c|c|c|c|c|}
\hline \multicolumn{19}{|c|}{ Sélection des plus importantes (i.e. deux premières) sources de connaissance actuelles (Act.) et idéales (Idéal) (\% de réponses) } \\
\hline \multirow[t]{2}{*}{$\begin{array}{c}\text { Sources de } \\
\text { connaissance }\end{array}$} & \multicolumn{3}{|c|}{$\begin{array}{l}\text { Qui suis-je } \\
\text { (entraineur) }\end{array}$} & \multicolumn{3}{|c|}{ Sélection de joueurs } & \multicolumn{3}{|c|}{$\begin{array}{c}\text { Plan annuel } \\
\text { d'entrainement }\end{array}$} & \multicolumn{3}{|c|}{$\begin{array}{c}\text { Habiletés techniques } \\
\text { et tactiques }\end{array}$} & \multicolumn{3}{|c|}{$\begin{array}{c}\text { Habiletés } \\
\text { psychologiques }\end{array}$} & \multicolumn{3}{|c|}{$\begin{array}{c}\text { Compétition } \\
\text { avant/pendant/après }\end{array}$} \\
\hline & Act. & Idéal & Dif. & Act. & Idéal & Dif. & Act. & Idéal & Dif. & Act. & Idéal & Dif. & Act. & Idéal & Dif. & Act. & Idéal & Dif. \\
\hline Expérience - athlète & 42 & 33 & & 33 & 17 & -16 & 58 & 17 & -41 & 75 & 42 & -33 & 75 & 25 & $-\mathbf{5 0}$ & 83 & 17 & -66 \\
\hline Expérience - entraineur & 67 & 67 & & 75 & 92 & 17 & 67 & 67 & & 92 & 75 & -17 & 42 & 42 & & 75 & 50 & -25 \\
\hline Certification en sport & 33 & 75 & 52 & 0 & 58 & 58 & 37 & 75 & 38 & 8 & 50 & 42 & 17 & 75 & 58 & 8 & 67 & 59 \\
\hline Conférences, séminaires & 0 & 17 & 17 & 8 & 0 & & 0 & 8 & & 0 & 17 & 17 & 8 & 17 & & 0 & 17 & 17 \\
\hline Mentor & 17 & 8 & & 0 & 0 & & 25 & 8 & -17 & 17 & 0 & -17 & 58 & 17 & -41 & 8 & 17 & \\
\hline Autres entraineurs & 33 & 0 & -33 & 42 & 8 & -34 & 8 & 17 & 11 & 8 & 8 & & 0 & 0 & & 0 & 0 & \\
\hline Livres/Vidéos & 8 & 0 & & 0 & 0 & & 8 & 0 & & 0 & 0 & & 0 & 0 & & 8 & 0 & \\
\hline Internet & 0 & 0 & & 0 & 0 & & 0 & 0 & & 0 & 0 & & 0 & 0 & & 0 & 0 & \\
\hline Journal/Carte réflexive & 0 & 0 & & 8 & 8 & & 0 & 8 & & 0 & 8 & & 0 & 25 & 25 & 0 & 17 & 17 \\
\hline Autre & 0 & 0 & & 0 & 0 & & 0 & 0 & & 0 & 0 & & 0 & 0 & & 0 & 0 & \\
\hline
\end{tabular}

\section{Discussion}

Globalement, les résultats de cette étude avec des entraineurs belges francophones confirment ceux obtenus avec des entraineurs chinois (He et al., 2018), canadiens (Erickson, Bruner, MacDonald, \& Côté, 2008; MacDonald, Beck, Erickson, \& Côté, 2016; McMaster, Culver, \& Werthner, 2012), portugais (Mesquita, Isidro, \& Rosado, 2010; Mesquita, Ribeiro, Santos, \& Morgan, 2014), espagnols (Jiménez, Lorenzo, \& Ibáñez, 2009), irlandais (Sherwin, Campbell, \& Macintre, 2017), turcs (Kilic \& Ince, 2015) et de différents pays (Stoszkowski \& Collins, 2016). Nous retiendrons plus particulièrement que : (a) les entraineurs utilisent plusieurs sources de connaissance; (b) l'expérience comme athlète et l'apprentissage sur le terrain (Expérience-entraineur) sont généralement les plus importantes sources de connaissance, et; (c) les programmes de formation pourraient jouer un rôle plus important. De plus, puisque certaines sources de connaissance semblent favoriser davantage l'acquisition de certaines connaissances, les responsables de formation (administrateurs et instructeurs) devraient adopter une approche holistique du développement des entraineurs. Nous partageons donc l'opinion de Crisfield (2019) déclarant que ces acteurs « doivent devenir des experts du processus d'apprentissage, de l'intervention et de l'entrainement sportif » (Chapter 15, traduction libre).

Dans une perspective d'avancement des connaissances sur la formation des entraineurs, nous poursuivons la discussion en rapportant ce que la plus récente littérature mentionne 
sur chacune des sources de connaissance. Cette position vise à aider chercheurs et décideurs (issus des fédérations et des pouvoirs publics) à poser des choix éclairés car le danger avec la reconnaissance de plusieurs sources de connaissance est de noyer les entraineurs dans une panoplie d'activités d'apprentissage (Trudel, Culver, \& Werthner, 2013). Ce danger est d'autant plus réel que la globalisation et les avancées technologiques font que les connaissances évoluent rapidement, sont de plus en plus variées et accessibles (Jarvis, 2006; Trede \& McEwen, 2016) et que les programmes de formation se multiplient, notamment aux États-Unis (Zakrajsek, Thompson, \& Dieffenbach, 2015) et en Angleterre (Turner \& Nelson, 2009).

\subsection{Les programmes de formation menant à la certification}

Les programmes de formation des entraineurs sont une composante essentielle dans une démarche visant à faire reconnaitre l'entraineur comme un professionnel (Duffy et al., 2011; Taylor \& Garratt, 2013). Ils permettent en effet aux organisations (fédérations, clubs, etc.) d'assurer un certain contrôle sur qui est certifié et à quel niveau (Gano-Overway, 2019). Dans le but d'augmenter l'impact positif des programmes de formation, des chercheurs ont récemment fait des suggestions que nous pouvons regrouper en quatre catégories :

(1) II faudrait mieux identifier le profil des formateurs d'entraineurs, c'est-à-dire ceux qui développent les programmes, enseignent le contenu et évaluent la rétention ou la mise en application (Abraham, 2016; Crisfield, 2019). Cette information permettrait de mieux les sélectionner et leur offrir une formation adaptée à leurs besoins (Culver et al., 2019; Cushion, Griffiths, \& Armour, 2019; North, 2010).

(2) II faudrait que les entraineurs prennent un rôle plus actif pendant les formations (Nelson, Cushion, \& Potrac, 2013). Cependant, le passage de l'approche traditionnelle dite 'd'enseignement centré sur l'instructeur/contenu' à une approche 'd'enseignement centré sur l'apprenant' est souvent une étape difficile (Cullen, Harris, \& Hill, 2012; Hussain, Trudel, Patrick, \& Rossi, 2012). Les réticences peuvent venir autant des instructeurs que des administrateurs et des apprenants (Weimer, 2013). Un argument souvent évoqué dans le milieu du sport est que les formations de trop longue durée impliquent des investissements considérables (temps et argent), lesquels n'incitent pas les entraineurs à s'inscrire (Bolter et al., 2018). Bien que, d'un point de vue financier, il soit avantageux de regrouper le maximum d'entraineurs pendant quelques soirs/jours pour transmettre le maximum d'information, du point de vue du développement des entraineurs, cette manière de 
procéder est loin d'être une situation idéale (Roy et al., 2018; Trudel \& Trottier, 2019).

Suite à une revue de la littérature, Paquette et Trudel (2016) concluent que la majorité des critiques sur les programmes de formation pourrait être prise en compte si l'approche respectant les principes de 'l'enseignement centré sur l'apprenant' était mise en place. Pour y parvenir, il est possible de se référer à des recommandations faites par différents auteurs (Nelson, Cushion, Potrac \& Groom, 2014; Paquette \& Trudel, 2018a, b).

(3) Les avancées en technologie (Technology-enhanced learning) (Cushion \& Townsend, 2016) ont permis d'innover dans la manière de former les entraineurs. II existe maintenant des programmes de formation complètement en ligne (Driska, 2018; Mallett \& Dickens, 2009; Santos et al., 2019) et d'autres qui utilisent une méthode mixte combinant la présence en classe avec l'utilisation d'outils technologiques (ex.: blogs, logs, journal en ligne, podcast) (De Martin-Silva, Fonseca, Jones, Morgan, \& Mesquita, 2015; Driska \& Gould, 2014; Jones et al., 2015; Stoszkowski \& Collins, 2014). Suite à une revue de littérature, Cushion et Townsend (2019) concluent que les effets sur l'apprentissage ne sont pas nécessairement dus à la technologie en elle-même mais bien à la manière avec laquelle la technologie est utilisée. Les auteurs terminent en soulignant que la littérature sur les retombées positives est insuffisante.

(4) Les programmes de formation actuellement offerts peuvent rarement préparer les entraineurs de $\mathrm{HN}$ à faire face à la complexité du coaching et à la nécessité d'apprendre à innover dans leur contexte (Collins, Burke, Martindale, \& Cruickshank, 2015; Milistetd et al., 2018; Phelan \& Griffiths, 2018). Il est donc nécessaire d'aller au-delà des programmes traditionnels dans lesquels un contenu est enseigné pour résoudre des problèmes précis et supposés être des défis pour tous les entraineurs (Collins et al., 2015; Nelson et al., 2014). Pour que les entraineurs deviennent des spécialistes dans leur contexte d'entrainement, il est important de pouvoir les soutenir dans une démarche d'apprentissage qui mettra en premier plan la pratique réflexive (Dixon, Lee, \& Ghaye, 2012; Knowles, Borrie, \& Telfer, 2005) où la capacité à cerner un problème prime sur celle à résoudre ce problème (Gilbert \& Trudel, 2006). Les entraineurs de HN acceptent de dévoiler ce qu'ils doivent améliorer ou de prendre des risques en explorant d'autres manières de faire, uniquement si l'environnement leur permet de se sentir en sécurité. Récemment, afin de créer des moments propices de réflexion et de les guider dans leur réflexion, il fut suggéré de mettre un formateur personnel à la disposition d'entraineurs de HN (LEADERS, 2019; 
McCarthy \& Brady, 2018; Trudel et al., 2016). Parmi les études empiriques qui explorent cette piste, soulignons celles de Mouchet et Maso (2018) qui ont validé une démarche pour aider des entraineurs professionnels d'une équipe de rugby française à réfléchir sur leur travail lors des discours de mi-temps, de Milistetd et al. (2018) qui ont accompagné un entraineur professionnel de tennis brésilien pendant six mois et de Rodrigue et Trudel (2019) qui ont travaillé avec cinq entraineurs de niveau universitaire canadien pendant une période de 12 mois. Toutes ces études tendent à démontrer l'intérêt de cette approche.

\subsection{Conférences et séminaires}

Conscientes de l'importance pour les entraineurs de garder leurs connaissances à jour, plusieurs organisations sportives exigent que ceux-ci démontrent qu'ils participent régulièrement à des activités de développement continu (e.g., Santos et al., 2017). Les conférences et séminaires sont souvent des occasions pour accumuler des crédits de formation. Cependant, il faut reconnaitre que le décompte des crédits accumulés est uniquement un indice de participation et non d'apprentissage. Selon certains auteurs, ces activités peuvent être une perte de temps et d'argent si (a) il est pris pour acquis que ce qui est enseigné est appris; (b) il y a trop de différences dans le profil des participants; (c) l'instructeur ne semble pas crédible aux yeux des participants; (d) les entraineurs ont le sentiment que ce qui est enseigné ne s'applique pas à leur contexte d'entrainement, et; (e) le contenu présenté n'a pas un impact visible sur leur travail (Moon, 2001; Roy et al., 2018; Trudel et al. 2013; Turnnidge \& Côté, 2017). Dans cette perspective, certaines fédérations s'appuient sur des pratiques réflexives et/ou la mise en place d'un suivi. Ceci présente un lien évident avec la littérature relative à la formation continue des enseignants en éducation physique.

\subsection{Mentor}

L'accès à un mentor semble très important lors des premières années dans un poste d'entraineur (Fairhurst, Bloom, \& Harvey, 2017; Young, 2013). Or, identifier cette personne ressource peut parfois être difficile et, pour cette raison, certains auteurs suggèrent de mettre en place des programmes formels de mentorat (Erickson, Côté, \& Fraser-Thomas, 2007; Jones, Harris, \& Miles, 2009; Mallett, Rynne, \& Billett, 2016). Bien que l'impact positif du mentorat ait été mentionné à plusieurs reprises (Bloom, 2013; Crips, 2018; White, Schempp, McCullick, Berger, \& Elliott, 2017), celui-ci pourrait être une limite au développement s'il devient un outil pour consolider les mêmes manières d'entrainer 
(Olsson, Cruickshank, \& Collins, 2017) ou devient une forme de contrôle social (Sawiuk, Taylor, \& Groom, 2018; Zehntner \& McMahon, 2019). De même, il semble essentiel de bien choisir les personnes qui peuvent jouer le rôle de mentor et ensuite leur offrir un programme de formation adapté aux besoins spécifiques à cette fonction (Bloom, 2013). A nouveau, il serait opportun de s'inspirer des études qui ont été conduites dans le domaine de la supervision et du mentorat des futurs enseignants en éducation physique.

\subsection{Autres entraineurs}

Chez les entraineurs sportifs, le partage de connaissances entre collègues est reconnu comme étant un moyen de développement (Mallett, 2010). Malheureusement, l'aspect compétitif inhérent au milieu amène certains entraineurs à limiter leurs interactions et à évoluer dans un environnement familier composé presque uniquement de leurs assistantsentraineurs (Lemire, Trudel, \& Durand-Bush, 2007; Trudel \& Gilbert, 2004). Dans le but de favoriser les interactions entre les entraineurs, des chercheurs ont proposé la création de 'communautés de pratique' (Culver \& Trudel, 2006; Mallett, 2010). Certaines études ont démontré que la mise en place de telles communautés était vue favorablement par les entraineurs (e.g., Bertram, Culver, \& Gilbert, 2016). Leur succès dépend souvent des qualités du facilitateur, ce qui amène à considérer que le retrait de ce dernier risque généralement de conduire à la disparition des activités (Gilbert, Gallimore, \& Trudel, 2009).

\subsection{Livres/Vidéos et Internet}

Tel que mentionné antérieurement, nous vivons dans une ère où l'information est abondante et facilement disponible. Selon Swora (2017), cette abondance amène avec elle un problème de sélection car il s'avère particulièrement difficile de vérifier la qualité des sources. Peu de gens ont d'ailleurs le temps de le faire. C'est ce qui ressort d'études réalisées auprès d'entraineurs à la recherche d'informations pratiques et scientifiques sur plusieurs plateformes (Google, You Tube, Facebook, etc.) (Koh, Lee, \& Lim, 2018; Kubayi, Coopoo, \& Toriola, 2019; Murray, 2011; Pope et al., 2015). Pour faciliter le transfert de connaissance entre cette gigantesque bibliothèque qu'est devenu Internet et des entraineurs en préparation physique, Szedlak et ses collègues (2019) ont validé avec succès l'utilisation de courtes vignettes disponibles en trois formats : écrites (environ 2000 mots), audio et vidéo (5 minutes ou moins). Les structures chargées de la formation des entraineurs devraient s'en inspirer. A ce niveau, il s'avère certainement utile d'assurer une mutualisation des ressources, notamment lorsqu'il s'agit d'explorer les documents disponibles dans d'autres langues. 


\subsection{Journal/Carte réflexive}

L'importance de la pratique réflexive pour le développement des entraineurs a été soulignée à plusieurs reprises (Gallimore, Gilbert, \& Nater, 2014; Knowles, Katz, \& Gilbourne, 2012). Cependant, bien qu'ils pensent continuellement à leur pratique, les entraineurs éprouvent des difficultés à s'engager dans une réflexion critique (Lara-Bercial \& Mallett, 2016). II importe de faire la différence entre penser à son coaching, ruminer des évènements spécifiques et faire une réflexion critique (Rynne \& Mallett, 2014; Werthner \& Trudel, 2009). De manière à aider les entraineurs dans leur pratique réflexive, il semble essentiel de leur proposer des outils (Cropley, Miles, \& Nichols, 2016; Trudel, Milistetd, \& Culver, 2020). Le journal de bord fait partie de ceux qui sont recommandés. Toutefois, bien que les entraineurs semblent reconnaitre son utilité lorsqu'il est proposé, il est rapidement abandonné lorsque non exigé (Knowles, Tyler, Gilbourne, \& Eubank, 2006). La principale raison évoquée pour expliquer cette situation fait référence au manque de temps. Par conséquent, de nouvelles initiatives qui prennent en compte les besoins et contextes des praticiens doivent être proposées et validées auprès des entraineurs. Récemment, Rodrigue et Trudel (2018) ont expliqué la démarche suivie par un entraineur de niveau universitaire pour développer sa propre carte réflexive reposant sur un nombre restreint de questions pertinentes, lesquelles devaient être répondues en moins de trois minutes après le match ou la séance d'entrainement.

\section{Conclusion}

Les résultats de cette étude à propos des sources de connaissance d'entraineurs belges francophones corroborent ceux obtenus auprès d'entraineurs de plusieurs pays localisés sur plusieurs continents. Ce que l'étude apporte de plus, c'est que les entraineurs font appel à des sources de connaissance différentes en fonction de la tâche qu'ils souhaitent maîtriser ou améliorer. Par conséquent, les responsables de formation ne peuvent se contenter d'offrir des programmes formels. Le menu d'activités d'apprentissage doit être varié et, surtout, adapté aux caractéristiques des entraineurs. Si on regarde vers le futur, les questions à se poser sont: (a) Qui seront les futurs entraineurs? (b) Quels seront leurs besoins? et (c) Quelles seront leurs préférences en termes de sources d'information? Pour y répondre, il conviendrait de diversifier les études portant sur la formation des entraineurs en évitant de se limiter à la littérature passée sur la formation des entraineurs mais bien en s'intéressant à une littérature prospective avec laquelle les chercheurs ne cherchent pas à prévoir l'avenir mais bien à proposer des scénarios possibles (Inayatullah, 2013; Monda, 
2018). Comme le soulignaient Cloes, Lenzen et Trudel (2009), il s'avérerait également fondamental de s'intéresser à la manière avec laquelle les résultats des recherches percolent vers les structures sportives et amènent une réelle transformation de la réalité de terrain. La recherche peut être féconde en termes de publications, son utilité ne sera amplifiée que lorsque les praticiens auront pu s'approprier les résultats et mis en pratique leurs implications.

\section{Bibliographie}

Abraham, A. (2016). Task analysis of coach developers: Applications to the FA youth coach educator role. In W. Allison, A. Abraham, \& A. Cale (Eds.), Advances in coach education and development: From research to practice (pp. 53-65). Oxon, UK: Routledge.

Araya, J., Bennie, A., \& O'Connor, D. (2015). Understanding performance coach development: Perceptions about a postgraduate coach education program. International Sport Coaching Journal, 2(1), 3-14.

Beaudoin, C., Callary, B., \& Trudeau, F. (2015). Coaches' adoption and implementation of Sport Canada's long-term athlete development model. SAGE Open, 5(3), 1-16.

Bennie, A., \& O'Connor, D. (2011). An effective coaching model: The perceptions and strategies of professional team sport coaches and players in Australia. International Journal of Sport and Health Science, 9, 98-104.

Bertram, R., Culver, D., \& Gilbert, W. (2016). Creating value in a sport coach community of practice: A collaborative inquiry. International Sport Coaching Journal, 3(1), 2-16.

Bloom, G.A. (2013). Mentoring for sport coaches. In P. Potrac, W. Gilbert, \& J. Denison (Eds.), Routledge handbook of sports coaching (pp. 476- 485). London: Routledge.

Bolter, N.D., Petranek, L.J., \& Dorsch, T.E. (2018). Coach, parent, and administrator perspectives on required coaching education in organized youth sport. International Journal of Sports Science \& Coaching, 13(3), 362-372.

Callary, B., Culver, D., Werthner, P., \& Bales, J. (2014). An overview of seven national high performance coach education programs. International Sport Coaching Journal, 1(3), 152- 164.

Capstick, A.L., \& Trudel, P. (2010). Coach communication of non-selection in youth competitive sport. International Journal of Coaching Science, 4(1), 3-23.

Carter, A.D., \& Bloom, G.A. (2009). Coaching knowledge and success: Going beyond athletic experiences. Journal of Sport Behavior, 32(4), 419-437. 
Cloes, M. (2012). La Wallonie en mouvement : vers une culture sportive et de l'activité physique ? Dans M. Germain et R. Robaye (Eds.), L'état de la Wallonie. Portrait d'un pays et de ses habitants - 2011 (pp. 70-80). Namur : Les éditions namuroises. Accessible sur Internet : http://hdl.handle.net/2268/117294

Cloes, M., Lenzen, B., \& Trudel, P. (2009). Analyse de la littérature francophone portant sur l'intervention de l'entraîneur sportif, publiée entre 1988 et 2007. STAPS : Revue Internationale des Sciences du Sport et de l'Éducation Physique, 30(1), 7-23. Accessible sur Internet : http://hdl.handle.net/2268/9229

Collins, D., Burke, V., Martindale, A., \& Cruickshank, A. (2015). The illusion of competency versus the desirability of expertise: Seeking a common standard for support professions in sport. Sports Medicine, 45(1), 1-7.

Côté, J. (2006). The development of coaching knowledge. International Journal of Sports Science \& Coaching, 1(3), 217-222.

Côté, J., Lidor, R., \& Hackfort, D. (2009). ISSP position stand: To sample or to specialize? Seven postulates about youth sport activities that lead to continued participation and elite performance. International Journal of Sport and Exercise Psychology, 7, 7-17.

Côté, J., Salmela, J., Trudel, P., Baria, A., \& Russell, S. (1995). The coaching model: A grounded assessment of expert gymnastic coaches' knowledge. Journal of Sport and Exercise Psychology, 17(1), 1-17.

Crisfield, P. (2019). Long-term coach development process. In K. Dieffenbach, \& M. Thompson (Eds.), Coach education essentials (Chapter 15). Champaign, IL: Human Kinetics.

Crisp, P. (2018). Sports coach mentoring-impacts on the mentors, not the 'mentees'. A case study of the Active Sussex Coach Support Officers Scheme. The Sport Journal, 19, $1-16$.

Cropley, B., Miles, A., \& Nichols, T. (2016). Learning to learn: The coach as a reflective practitioner. In J. Wallis \& J. Lambert (Eds.), Becoming a sports coach (pp. 11-25). London: Routledge.

Cullen, R., Harris, M., \& Hill, R. (2012). The learner-centred curriculum: Design and implementation. San Francisco, CA: Jossey-Bass.

Culver, D., \& Trudel, P. (2006). Cultivating coaches' communities of practice: Developing the potential for learning through interactions. In R.L. Jones (Ed.), The sports coach as educator: Re-conceptualising sports coaching (pp. 97-112). Abingdon: Routledge. 
Culver, D., Werthner, P., \& Trudel, P. (2019). Coach developers as 'facilitators of learning' in a large-scale coach education programme: One actor in a complex system. International Sport Coaching Journal, 6(3), 1-12.

Cushion, C., Griffiths, M., \& Armour, K. (2019). Professional coach educators in-situ: A social analysis of practice. Sport, Education and Society, 24(5), 533-546.

Cushion C., Nelson L., Armour K., Lyle, J., Jones, R., Sandford, R., \& O'Callaghan, C. (2010). Coach learning and development: A review of literature. London, UK: The National Coaching Foundation.

Cushion, C., \& Townsend, R.C. (2019). Technology-enhanced learning in coaching: A review of literature. Educational Review, 71(5), 631-649.

De Martin-Silva, L., Fonseca, J., Jones, R. L., Morgan, K., \& Mesquita, I. (2015). Understanding undergraduate sports coaching students' development and learning: the necessity of uncertainty. Teaching in Higher Education, 20(7), 669-683.

Din, C., \& Paskevich, D. (2013). An integrated research model of Olympic podium performance. International Journal of Sports Science \& Coaching, 8(2), 431-444.

Dixon, M., Lee, S., \& Ghaye, T. (2012). Coaching for performance: An interview with Olympic diving coach, Andy Banks. Reflective Practice, 13(3), 339-354.

Driska, A.P. (2018). A Formative, Utilization-Focused Evaluation of USA Swimming's Nationwide Online Coach Education Program. International Sport Coaching Journal, 5(3), 261-272.

Driska, A.P., \& Gould, D. (2014). Evaluating a problem-based group learning strategy for online, graduate-level coach education. Kinesiology Review, 3(4), 227-234.

Duarte, T., \& Culver, D. (2014). Becoming a coach in developmental adaptive sailing: A lifelong learning perspective. Journal of Applied Sport Psychology, 26(4), 441-456.

Duffy, P., Hartley, H., Bales, J., Crespo, M., Dick, F., Vardhan, D., et al. (2011). Sport coaching as a 'profession': Challenges and future directions. Journal of Coaching Science, 5(2), 93- 124.

Erickson, K., Bruner, M. W., MacDonald, D. J., \& Côté, J. (2008). Gaining insight into actual and preferred sources of coaching knowledge. International Journal of Sports Science \& Coaching, 3(4), 527-538.

Erickson, K., Côté, J., \& Fraser-Thomas, J. (2007). Sport experiences, milestones, and educational activities associated with high-performance coaches' development. The Sport Psychologist, 21(3), 302-316.

Fairhurst, K.E., Bloom, G.A., \& Harvey, W.J. (2017). The learning and mentoring experiences of Paralympic coaches. Disability and Health Journal, 10(2), 240-246. 
Gallimore, R., Gilbert, W., \& Nater, S. (2014). Reflective practice and ongoing learning: A coach's 10-year journey. Reflective Practice, 15(2), 268-288.

Gano-Overway, L.A. (2019). Reflection on accreditation and endorsement of coach education and development programs. In K. Dieffenbach, \& M. Thompson (Eds.), Coach education essentials (Chapter 16). Champaign, IL: Human Kinetics.

Gilbert, W., Gallimore, R., \& Trudel, P. (2009). A learning community approach to coach development in youth sport. Journal of Coaching Education, 2(2), 1-21.

Gilbert, W., \& Trudel, P. (2006). The coach as a reflective practitioner. In R. Jones (Ed.), The sports coach as educator: Re-conceptualising sports coaching (pp. 113-127). Abingdon: Routledge.

Hamilton, M.G.B., \& LaVoi, N.M. (2019). Coaches who care: Moral exemplars in collegiate athletics. Journal of Applied Sport Psychology, 32(1), 81-103.

He, C., Trudel, P., \& Culver, D. (2018). Actual and ideal sources of coaching knowledge of elite Chinese coaches. International Journal of Sports Science \& Coaching, 13(4), 496-507.

Hussain, A., Trudel, P., Patrick, T., \& Rossi, A. (2012). Reflections on a novel coach education program: A narrative analysis. International Journal of Sports Science \& Coaching, 7(2), 227-240.

Inayatullah, S. (2013). Futures studies: Theories and methods. In F.G. Junquera (Ed.), There's a future: Visions for a better world (pp. 36-66). Madrid: BBVA.

Issurin, V.B. (2010). New horizons for the methodology and physiology of training periodization. Sports Medicine, 40(3), 189-206.

Jiménez, S., Lorenzo, A., \& Ibáñez, S. (2009). Development of expertise in Spanish elite basketball coaches. Revista Internacional de Ciencias del Deporte, 17, 19-32.

Jarvis, P. (2006) Towards a comprehensive theory of learning, London: Routledge.

Jones, R.L., Fonseca, J., De Martin Silva, L., Davies, G., Morgan, K., \& Mesquita, I. (2015). The promise and problems of video diaries: building on current research. Qualitative Research in Sport, Exercise and Health, 7(3), 395-410.

Jones, R.L., Harris, R.A., \& Miles, A. (2009). Mentoring in sports coaching: A review of the literature. Physical Education and Sport Pedagogy, 14(3), 267-284.

Jones, R.L., \& Wallace, M. (2005). Another bad day at the training ground: Coping with ambiguity in the coaching context. Sport Education and Society, 10(1), 119-134.

Kilic, K., \& Ince, M. L. (2015). Use of sports science knowledge by Turkish coaches. International Journal of Exercise Science, 8(1), 21. 
Knowles, Z, Borrie, A., \& Telfer, H. (2005). Towards the reflective sports coach: Issues of context, education and application. Ergonomics, 48(11-14), 1711-1720.

Knowles, Z., Katz, J., \& Gilbourne, D. (2012). Reflective practice within elite consultancy: Diary extracts and further discussion on a personal and elusive process. The Sport Psychologist, 26(3), 454-469.

Knowles, Z., Tyler, G., Gilbourne, D. \& Eubank, M. (2006). Reflecting on reflection: Exploring the practice of sports coaching graduates. Reflective Practice, 7(2), 163-179.

Koh, K.T., Lee, T.P., \& Lim, S.H. (2018). The Internet as a source of learning for youth soccer coaches. International Journal of Sports Science \& Coaching, 13(2), 278289.

Koh, K.T., Mallett, C., Camiré, M., \& Wang, C.K.J. (2015). A guided reflection intervention for high performance basketball coaches. International Sport Coaching Journal, 2(3), 273-284.

Kristiansen, E., \& Roberts, G. C. (2010). Young elite athletes and social support: Coping with competitive and organizational stress in "Olympic" competition. Scandinavian Journal of Medicine \& Science in Sports, 20(4), 686-695.

Kubayi, A., Coopoo, Y., \& Toriola, A. (2019). Knowledge transfer from sport science to coaching: A South African coach's perspective. South African Journal for Research in Sport, Physical Education and Recreation, 41(1), 51-61.

Kuklick, C.R., Gearity, B.T., \& Thompson, M. (2015). Reflective practice in a universitybased coach education program. International Sport Coaching Journal, 2(3), 248260.

Lara-Bercial, S., \& Mallett, C. (2016). The practices and developmental pathways of professional and Olympic serial winning coaches. International Sport Coaching Journal, 3(3), 221-239.

Leaders Performance Institute [LEADERS]. (2019). Coaching the coaches - Coach education and development: The state of play across elite sport. London, UK: David Cushnan.

Lemyre, F., Trudel, P., \& Durand-Bush, N. (2007). How youth-sport coaches learn to coach. The Sport Psychologist, 21(2), 191-209.

MacDonald, D.J., Beck, K., Erickson, K., \& Côté, J. (2016). Understanding sources of knowledge for coaches of athletes with intellectual disabilities. Journal of Applied Research in Intellectual Disabilities, 29(3), 242-249. 
Mallett, C. (2010). Becoming a high-performance coach: Pathways and communities. In J. Lyle \& C. Cushion (Eds.), Sports coaching: Professionalism and practice (pp. 119134). London: Elsevier.

Mallet, C. \& Dickens, S. (2009). Authenticity in formal coach education: Online postgraduate studies in sports coaching at the University of Queensland. International Journal of Coaching Science, 3(2), 79-90.

Mallett, C., Rynne, S., \& Billett, S. (2016). Valued learning experiences of early career and experienced high-performance coaches. Physical Education and Sport Pedagogy, 21(1), 89-104.

McCarthy, L., \& Brady, A. (2018). Coaching the coaches: Appreciative reflection and appreciative inquiry in the development of sport coaches. In A. Brady \& B. GrenvilleCleave (Eds.), Positive psychology in sport and physical activity: An introduction (pp. 232-242). Oxon, UK: Routledge.

McMaster, S., Culver, D., \& Werthner, P. (2012). Coaches of athletes with a physical disability: a look at their learning experiences. Qualitative Research in Sport, Exercise and Health, 4(2), 226-243.

Mesquita, I., Isidro, S., \& Rosado, A. (2010). Portuguese coaches' perceptions of and preferences for knowledge sources related to their professional background. Journal of Sports Science \& Medicine, 9(3), 480-489.

Mesquita, I., Ribeiro, J., Santos, S., \& Morgan, K. (2014). Coach learning and coach education: Portuguese expert coaches' perspective. The Sport Psychologist, 28(2), 124-136.

Milistetd, M., Peniza, L., Trudel, P., \& Paquette, K. (2018). Nurturing High-Performance Sport Coaches' Learning and Development using a Narrative-Collaborative Coaching Approach. LASE Journal of Sport Science, 9(1), 6-38.

Monda, E. (2018). Social futuring - In the context of futures studies. Society and Economy, 40(s1), 77-109.

Moon, J. (2001). Short courses \& workshops: Improving the impact of learning, training \& professional development. London: Kogan Page.

Mouchet, A., \& Maso, F. (2018). Subjective lived experience: A resource for coaches' education. LASE Journal of Sport Science, 9(1), 60-77.

Murray, M. (2011). Delivering coaching education online: Practical tips for online educators. Journal of Coaching Education, 4(2), 62-72.

Nash, C., \& Sproule, J. (2012). Coaches perceptions of their coach education experiences. International Journal of Sport Psychology, 43(1), 33. 
Nelson, L., Cushion, C., \& Potrac, P. (2013). Enhancing the provision of coach education: the recommendations of UK coaching practitioners. Physical Education and Sport Pedagogy, 18(2), 204-218.

Nelson, L., Cushion, C. J., Potrac, P., \& Groom, R. (2014). Carl Rogers, learning and educational practice: Critical considerations and applications in sports coaching. Sport, Education and Society, 19(5), 513-531.

North, J. (2010). Using 'coach developers' to facilitate coaching learning and development: Qualitative evidence from UK. International Journal of Sports Science \& Coaching, 5(2), 239-256.

Olsson, C. Cruickshank, A., \& Collins, D. (2017). Making mentoring work: The need for rewiring epistemology. Quest, 69(1), 50-64.

Olusoga, P., Butt, J., Maynard, I., \& Hays, K. (2010). Stress and coping: A study of worldclass coaches. Journal of Applied Sport Psychology, 22(3), 274-293.

Paquette, K., \& Trudel, P. (2016). Learner-centered teaching: A consideration for revitalizing coach education. In P. A. Davis (Ed.), The psychology of effective coaching and management (pp. 53-70). New York: Nova Science.

Paquette, K., \& Trudel, P. (2018a). The evolution and learner-centered status of a coach education program. International Sport Coaching Journal, 5(1), 24-36.

Paquette, K., \& Trudel, P. (2018b). Learner-centered coach education: Practical recommendations for coach development administrators. International Sport Coaching Journal, 5(2), 169-175.

Paquette, K., Trudel, P., Duarte, T., \& Cundari, G. (2019). Participating in a learner-centered coach education program: Composite vignettes of coaches' and coach educators' experiences. International Sport Coaching Journal, 6(3), 274-284.

Phelan, S., \& Griffiths, M. (2019). Reconceptualising professional learning through knowingin-practice: A case study of a coaches high performance centre. Sports Coaching Review, 8(2), 103-123.

Piggott, D. (2012). Coaches' experiences of formal coach education: A critical sociological investigation. Sport, Education and Society, 17(4), 535-554.

Pope, J.P., Stewart, N.W., Law, B., Hall, C.R., Gregg, M.J., \& Robertson, R. (2015). Knowledge translation of sport psychology to coaches: Coaches' use of online resources. International Journal of Sports Science \& Coaching, 10(6), 1055-1070.

Roberts, C.M., \& Faull, A.L. (2013). Building a successful Olympic team selection protocol in women's handball: A case study examining the benefits of employing reflective practice. Reflective Practice, 14(5), 648-659. 
Rodrigue, F., \& Trudel, P. (2018). Reflective practice: A case study of a university football coach using reflective cards. LASE Journal of Sport Science, 9(1), 39-59.

Rodrigue, F., Trudel, P. (2019). A 'personal learning coach' for high-performance coaches: A companion to reflect and learn from one's own coaching practice. In B. Gearity \& B. Callary (Eds.), Coach education and development in sport: Instructional strategies (pp. 141-153). London: Routledge.

Roy, M., Roy, X., Chevrier, J., \& Cardinal, C. (2018). Planning and monitoring of sports training: What is it and how to teach it? LASE Journal of Sport Science, 9(1), 91123.

Rynne, S., \& Mallett, C. (2014). Coaches' learning and sustainability in high performance sport. Reflective Practice, 15(1), 12-26.

Santos, F., Camiré, M., MacDonald, D. J., Campos, H., Conceição, M., \& Silva, P. (2017). Youth sport coaches' perspective on positive youth development and its worth in mainstream coach education courses. International Sport Coaching Journal, 4(1), 38-46.

Santos, F., Camiré, M., MacDonald, D. J., Campos, H., Conceição, M., \& Silva, A. (2019). Process and outcome evaluation of a positive youth development-focused online coach education course. International Sport Coaching Journal, 6(1), 1-12.

Sawiuk, R., Taylor, W. G., \& Groom, R. (2018). Exploring formalized elite coach mentoring programmes in the UK: 'We've had to play the game'. Sport, Education and Society, 23(6), 619-631.

Sherwin, I., Campbell, M.J., \& Macintyre, T.E. (2017). Talent development of high performance coaches in team sports in Ireland. European Journal of Sport Science, 17(3), 271-278.

Stoszkowski, J., \& Collins, D. (2014). Blogs: A tool to facilitate reflection and community of practice in sports coaching? International Sport Coaching Journal, 1, 130-151.

Stoszkowski, J., \& Collins, D. (2016). Sources, topics and use of knowledge by coaches. Journal of Sports Sciences, 34(9), 794-802.

Swora, C. (2017). Rules of engagement: Building a workplace culture to thrive in an uncertain world. Published by C. Swora and Pinnacle Culture.

Szedlak, C., Smith, M.J., Callary, B., \& Day, M.C. (2019). Using written, audio, and video vignettes to translate knowledge to elite strength and conditioning coaches. International Sport Coaching Journal, 6(2) 199-210. 
Taylor, W.G., \& Garratt, D. (2013). Coaching and professionalization. In P. Potrac, W. Gilbert, \& J. (Eds.), Routledge handbook of sports coaching (pp. 27-39). London: Routledge.

Thelwell, R.C., Weston, N.J., Greenlees, I.A., \& Hutchings, N.V. (2008). A qualitative exploration of psychological-skills use in coaches. The Sport Psychologist, 22(1), 38-53.

Trede, F., \& McEwen, C. (2016). Scoping the deliberate professional. In F. Trede \& C. McEwen (Eds.), Educating the deliberate professional: Preparing for future practices (pp. 3-14). New York: Springer.

Trudel, P., Culver, D., \& Richard, J.P. (2016). Peter Jarvis: Lifelong learning. In L. Nelson, R. Groom, \& P. Potrac (Eds.), Learning in sports coaching: Theory and application (pp. 202-214). London, UK: Routledge.

Trudel, P., Culver, D., \& Werthner, P. (2013). Looking at coach development from the coach-learner's perspective: Consideration for coach development administrators. In P. Potrac, W. Gilbert, \& J. (Eds.), Routledge handbook of sports coaching (pp. 375-387). London: Routledge.

Trudel, P., \& Gilbert, W. (2004). Communities of practice as an approach to foster ice hockey coach development. In D. J. Pearsall \& A. B. Ashare (Eds.), Safety in ice hockey: Fourth Volume ASTM STP 1446 (pp. 167-179). West Conshohoken: ASTM International

Trudel, P., Gilbert, W., \& Rodrigue, F. (2016). The journey from competent to innovator: Using Appreciative Inquiry to enhance high performance coaching. Al Practitioner, 18(2), 40-46.

Trudel, P., Milestetd, M., \& Culver, D. (2020). What the empirical studies on sport coach education programs in higher education have to reveal: A review. International Sport Coaching Journal, 7(1), 61-73.

Trudel, P., \& Trottier, C. (2019). Learning environments for helping coaches and other key stakeholders include life skills in their practice In F. Santos, L. Strachan, P. Pereira, D. MacDonald, \& K. Erickson (Eds.), Coaching positive youth development: Implications and practices from around the world (pp. 101-117). Porto: School of Higher Education, Polytechnic Institute of Porto.

Turner, D., \& Nelson, L.J. (2009). Graduate perceptions of a UK university based coach education programme, and impacts on development and employability. International Journal of Coaching Science, 3(2), 3-28. 
Turnnidge, J., \& Côté, J. (2017). Transformational coaching workshop: Applying a personcentred approach to coach development programs. International Sport Coaching Journal, 4(3), 314-325.

Vargas-Tonsing, T.M. (2007). Coaches' preferences for continuing coaching education. International Journal of Sports Science \& Coaching, 2(1), 25-35.

Watts, D.W., \& Cushion, C. (2017). Coaching journeys: Longitudinal experiences form professional football in Great Britain. Sports Coaching Review, 6(1), 76-93.

Weimer, M. (2013). Learner-centred teaching: Five key changes to practice (2nd ed.). San Francisco, CA: Jossey-Bass.

Werthner, P., \& Trudel, P. (2009). Investigating the idiosyncratic learning paths of elite Canadian coaches. International Journal of Sports Science \& Coaching, 4(3), 433449.

White, J.S., Schempp, P.G., McCullick, B.A., Berger, B.S., \& Elliott, J.M. (2017). Mentoring relationships in sport from the protégé's perspective. International Journal of Evidence Based Coaching and Mentoring, 15(1), 152.

Young, B.W. (2013). Coaching expertise and the quantitative examination of developmental experiences. In P. Potrac, W. Gilbert \& J. Denison (Eds.), Routledge handbook of sports coaching (pp. 437-450). London: Routledge.

Young, B.W., \& Callary, B. (2018). Doing 'more for adult sport': Promotional and programmatic efforts to offset adults' psycho-social obstacles. In R.A. Dionigi \& M. Gard (Eds.), Sport and physical activity across the lifespan (pp. 263-282). London: Palgrave Macmillan.

Zakrajsek, R., Thompson, M., \& Dieffenbach, K. (2015). An exploration of the academic coaching education internship. Sports Coaching Review, 4(1), 24-40.

Zehntner, C., \& McMahon, J. A. (2014). Mentoring in coaching: the means of correct training? An autoethnographic exploration of one Australian swimming coach's experiences. Qualitative Research in Sport, Exercise and Health, 6(4), 596-616. 